\section{Drug-induced lupus erythematosus following immunotherapy with anti-programmed death- (ligand) 1}

We read with interest the study of Kostine et al describing rheumatic immune-related adverse events (irAE), which occur in $6.6 \%$ of patients treated for cancer by anti-programmed death-(ligand) 1 (PDL1). ${ }^{1}$ These new adverse effects pose significant challenges to patient care in terms of optimal management of these autoimmune damaging toxicities, while allowing effective antitumor therapy to continue.

The $\mathrm{PD}(\mathrm{L}) 1$ pathway is involved in the maintenance of immune tolerance, and the blockage of this axis by anticancer immunotherapy could trigger autoimmune diseases and especially lupus. ${ }^{2} 3$ We then searched in the pharmacovigilance register of our institution-the 'Registre des Effets Indésirables Sévères des Anticorps Monoclonaux Immunomodulateurs en Cancérologie (REISAMIC)'-whether cases of drug-induced lupus erythematosus (DI-LE) were reported following anti$\mathrm{PD}(\mathrm{L}) 1$ immunotherapies.

Between October 2013 and July 2017, five cases of DI-LE were recorded in REISAMIC. Given the number of patients having received anti-PD(L)1 during the same period $(n=1044)$, the estimated incidence of DI-LE was $0.48 \%$. All patients gave their written informed consent for the use of their data in this report. The patients' characteristics are summarised in table 1. The patients had developed DI-LE at a median (range) age of $63(48-80)$ years. None of the patients had a history of autoimmune disease before starting anti-PD(L)1. The most specific sign of DI-LE was subacute cutaneous lupus erythematous (SCLE) in four patients and chilblain lupus in the remaining patient. One patient having SCLE had also declared a systemic lupus erythematosus (SLE) according to the Systemic Lupus International Collaborating Clinics criteria. ${ }^{4}$ The DI-LE was revealed by a frank maculopapular rash in the four patients with SCLE (figure 1). The median time of DI-LE occurrence was 10 (range: 4-22) weeks after the initiation of immunotherapy. Antinuclear antibodies in serum were found positive for two (40\%) out of the five patients and were specifically positive for anti-Sjögren's syndrome-related antigen A (SSA). These two SSA-positive patients had SCLE but no eye or mouth dryness symptoms suggestive of Sjögren's disease. A skin biopsy was performed in all cases except the chilblain lupus. The skin biopsies revealed a lymphocytic infiltrate of the dermis, predominantly around adnexal sites. Alcian blue staining revealed mucin deposits in all patients. Direct immunofluorescence assays for IgG or C3 in skin biopsy were positive in two of the four patients tested (50\%). The treatment of DI-LE was based on topical corticosteroids in all cases, with the antimalarial hydroxychloroquine added in the SLE case, and the outcome was favourable with a resolution in all cases.

This report is the first series of cases of lupus erythematosus induced by anti-PD(L)1 immunotherapy. A recent similar case report of pembrolizumab-related subacute cutaneous lupus erythematosus was provided. ${ }^{5}$ The DI-LE has been variously reported after drug exposure such as hydralazine, procainamide, quinidine, oestrogen, tumour necrosis factor inhibitors, chlorpromazine, isoniazid, practolol, penicillamine and minocycline. ${ }^{6}$ We believe that anti-PD(L)1 immunotherapies should also now be added to this list.

Based on our experience and the present case series, DI-LE induced by anti-PD(L)1 was characterised by an extensive,

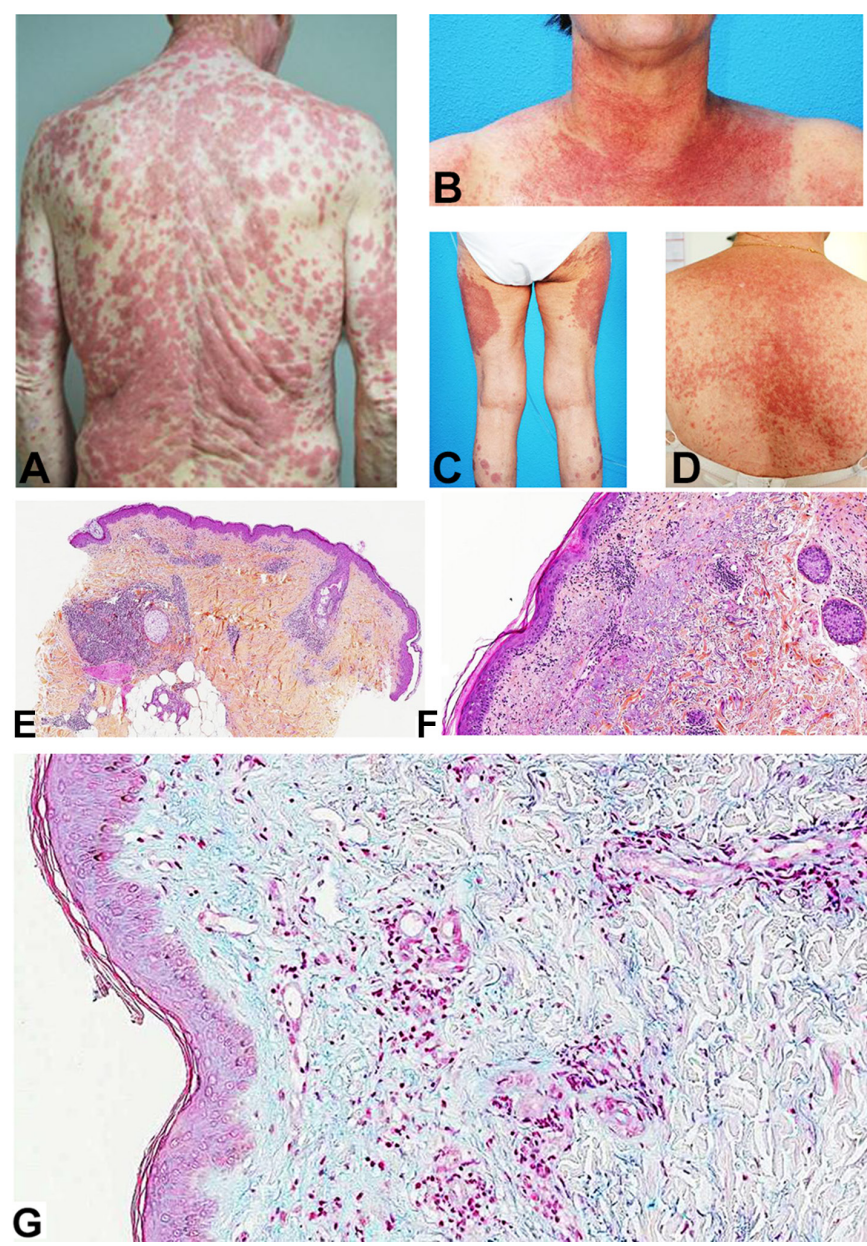

Figure 1 Photographs and histologic assessment of skin biopsies of cutaneous lupus erythematosus lesions induced by treatment with anti-PD(L)1. (A) Patient 4, erythematous papules and plaques with an annular, polycyclic configuration: generalised subacute lupus erythematous. (B) Patient 1, erythematous macules on the neck: subacute cutaneous lupus erythematosus. (C) Patient 2, symmetric papulosquamous erythematous rashes on the lower limbs. (D) Patient 3, erythematous macules and plaques on the back: subacute cutaneous lupus erythematosus. (E) Skin biopsy from patient 1, haematoxylin eosin saffron (HES) staining, $\times 2.5$ : peripheral and periadnexal monomorphic lymphocytic inflammatory infiltrate over the entire dermis. (F) Skin biopsy from patient 4 , HES staining, $\times 5$ : lichenoid dermatosis with staged apoptotic bodies in the epidermis. Peripheral inflammatory mononuclear infiltrate in the upper dermis. (G) Skin biopsy from patient 3 , Alcian blue staining, $\times 10$ : mucin deposits in the dermis.

non-itchy and frankly macular or papular erythematous rash. The DI-LE diagnosis relies on the combination of the dermatological presentation associated with pathological features characterised by a lymphocytic dermal infiltration predominantly located at periadnexal sites, and mucin deposits. ${ }^{7}$ The confrontation between the clinical appearance and the pathological aspects is often useful to differentiate between DI-LE and other non-specific cutaneous irAEs, or other specific autoimmune skin diseases that can be induced by anti-PD(L)1 such as psoriasis, toxic epidermal necrolysis, lichen planus, bullous dermatitis and dermatomyositis. $^{8}$

These new cases of lupus induced by anti-PD(L) 1 should incite rheumatologist and internists to dedicate further prospective study for irAE. Investigation of potential biomarkers of irAEs such as the genetic background, serum 


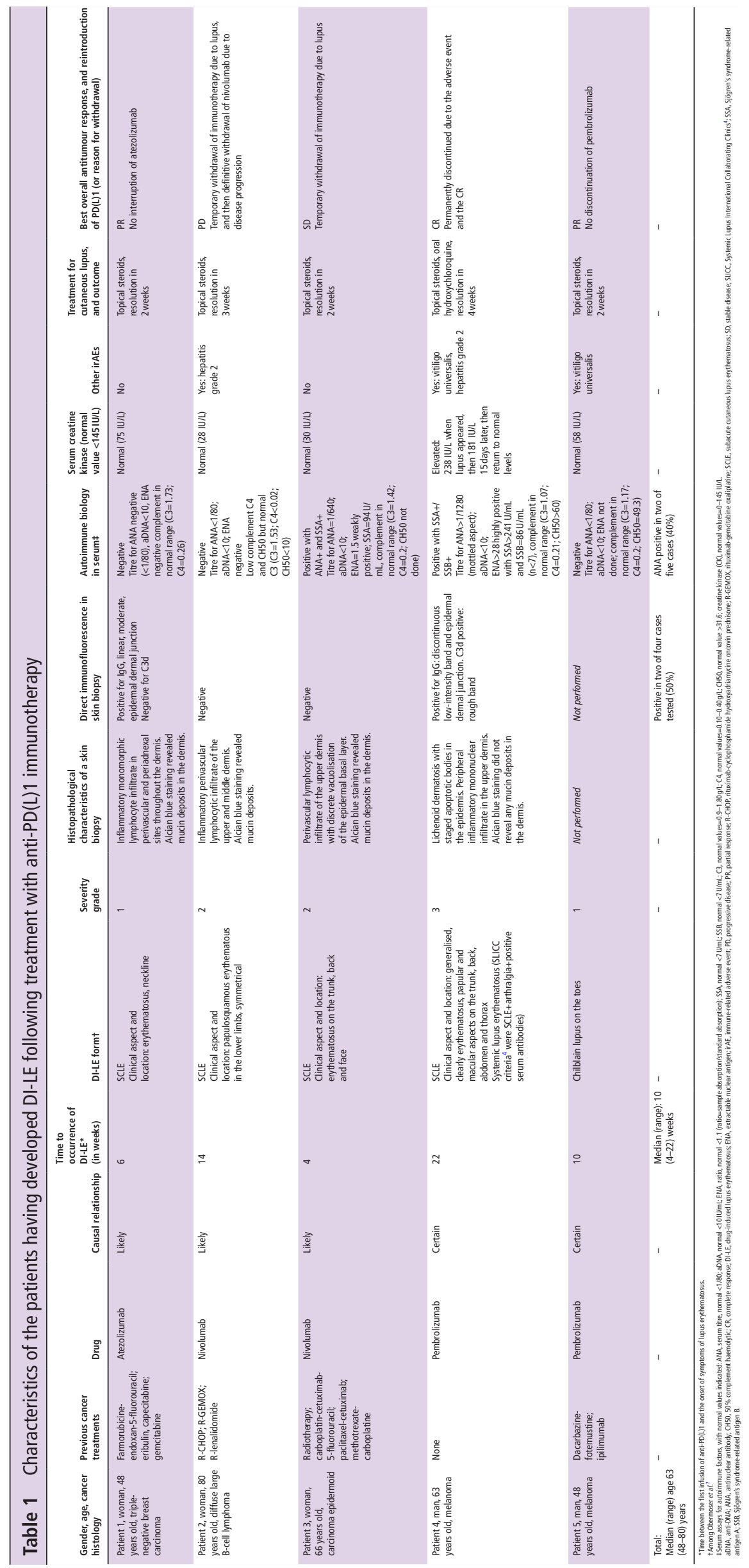




levels of autoimmune factors and cytokines may help better understand these immunological adverse events and autoimmune conditions in general.

Jean-Marie Michot, ${ }^{1,2}$ Mathilde Fusellier, ${ }^{1}$ Stephane Champiat, ${ }^{1,3}$ Charles Velter, ${ }^{4}$ Capucine Baldini, ${ }^{1,3}$ Anne-Laure Voisin, ${ }^{5}$

Francois-Xavier Danlos, ${ }^{1}$ Yolla El Dakdouki, ${ }^{1}$ Maxime Annereau, ${ }^{6}$ Xavier Mariette, ${ }^{7}$ Caroline Robert, ${ }^{4}$ Khadija Cherif, ${ }^{8}$ Aurélien Marabelle, ${ }^{1}$ Christine Mateus, ${ }^{4}$ Olivier Lambotte ${ }^{2,3,9,10}$

1 Medical Oncology and Drug Development Department, Institut Gustave Roussy, Villejuif, France

${ }^{2}$ Department of Internal Medicine and Clinical Immunology, Hôpital Bicêtre, Le Kremlin-Bicêtre, France

${ }^{3}$ University of Paris Sud, Le Kremlin-Bicêtre, France

${ }^{4}$ Department of Medical Oncology, Institut Gustave Roussy, Villejuif, France

${ }^{5}$ Department of Pharmacovigilance, Institut Gustave Roussy, Villejuif, France

${ }^{6}$ Department of Pharmacy, Institut Gustave Roussy, Villejuif, France

${ }^{7}$ Department of Rheumatology, Hôpital Bicêtre, Le Kremlin-Bicêtre, France

${ }^{8}$ Department of Biopathology, Institut Gustave Roussy, Villejuif, France

${ }^{9}$ INSERM U1184, Immunology of Viral Infections and Autoimmune Diseases, Le Kremlin- Bicêtre, France

${ }^{10} \mathrm{Commissariat} \mathrm{à} \mathrm{I'Energie} \mathrm{Atomique} \mathrm{(CEA),} \mathrm{Fontenay-aux-Roses,} \mathrm{France}$

Correspondence to Dr. Jean-Marie Michot, Drug Development Department, Institut Gustave Roussy, Villejuif F-94805, France;

jean-marie.michot@gustaveroussy.fr

Handling editor Josef S Smolen

Acknowledgements The authors thank David Fraser (Biotech Communication SARL, Ploudalmézeau, France) for copy-editing assistance.

Contributors All authors contributed to the patient care management and manuscript writing. All authors approved the manuscript submitted.

Competing interests None declared.

Patient consent Obtained.

Ethics approval Ethics Board Committee and Institutional Board of Institut Gustave Roussy.

Provenance and peer review Not commissioned; internally peer reviewed. (c) Article author(s) (or their employer(s) unless otherwise stated in the text of the article) 2019. All rights reserved. No commercial use is permitted unless otherwise expressly granted.

\section{A) Check for updates}

To cite Michot J-M, Fusellier M, Champiat S, et al. Ann Rheum Dis 2019;78:e67.

Received 29 April 2018

Accepted 1 May 2018

Published Online First 1 June 2018

\section{SLinked}

http://dx.doi.org/10.1136/annrheumdis-2018-213691

Ann Rheum Dis 2019:78:e67. doi:10.1136/annrheumdis-2018-213677

\section{REFERENCES}

1 Kostine M, Rouxel L, Barnetche T, et al. Rheumatic disorders associated with immune checkpoint inhibitors in patients with cancer-clinical aspects and relationship with tumour response: a single-centre prospective cohort study. Ann Rheum Dis 2018;77:393-8

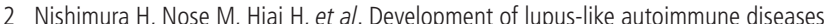
by disruption of the PD-1 gene encoding an ITIM motif-carrying immunoreceptor Immunity 1999:11:141-51.

3 Prokunina L, Castillejo-López C, Oberg F, et al. A regulatory polymorphism in PDCD1 is associated with susceptibility to systemic lupus erythematosus in humans. Nat Genet 2002:32:666-9.

4 Petri M, Orbai AM, Alarcón GS, et al. Derivation and validation of the Systemic Lupus International Collaborating Clinics classification criteria for systemic lupus erythematosus. Arthritis Rheum 2012;64:2677-86.

5 Shao K, McGettigan S, Elenitsas R, et al. Lupus-like cutaneous reaction following pembrolizumab: An immune-related adverse event associated with anti-PD-1 therapy. $J$ Cutan Pathol 2018:45:74-7.

6 Niklas K, Niklas AA, Majewski D, et al. Rheumatic diseases induced by drugs and environmental factors: the state-of-the-art - part two. Reumatologia 2016;54:165-9.

7 Obermoser G, Sontheimer RD, Zelger B. Overview of common, rare and atypical manifestations of cutaneous lupus erythematosus and histopathological correlates. Lupus 2010;19:1050-70.

8 Hofmann L, Forschner A, Loquai C, et al. Cutaneous, gastrointestinal, hepatic, endocrine, and renal side-effects of anti-PD-1 therapy. Eur J Cancer 2016:60:190-209. 